\title{
ILCEA
}

Revue de l'Institut des langues et cultures

d'Europe, Amérique, Afrique, Asie et Australie

$11 \mid 2009$

Langues \& cultures de spécialité à l'épreuve des médias

\section{Vladimir Poutine à l'épreuve du Corriere della Sera}

Restructuration du discours et construction d'image

\section{Elisa Rossi Danelzik}

\section{OpenEdition}

\section{Journals}

Édition électronique

URL : http://journals.openedition.org/ilcea/178

DOI : $10.4000 /$ ilcea. 178

ISSN : 2101-0609

Éditeur

UGA Éditions/Université Grenoble Alpes

Édition imprimée

ISBN : 978-2-84310-179-3

ISSN : $1639-6073$

Référence électronique

Elisa Rossi Danelzik, "Vladimir Poutine à l'épreuve du Corriere della Sera », ILCEA [En ligne], 11 | 2009, mis en ligne le 30 avril 2009, consulté le 08 mars 2021. URL : http://journals.openedition.org/ilcea/178 ; DOI : https://doi.org/10.4000/ilcea.178

Ce document a été généré automatiquement le 8 mars 2021

(c) ILCEA 


\title{
Vladimir Poutine à l'épreuve du Corriere della Sera
}

\author{
Restructuration du discours et construction d'image
}

Elisa Rossi Danelzik

1 Historiquement importants, les liens entre l'Italie et la Fédération de Russie sont aujourd'hui particulièrement forts, vu les relations commerciales intenses entre les deux pays et surtout les liens personnels existant entre leurs plus hauts responsables. La Russie est le premier fournisseur d'énergie pour l'Italie ; en Europe, la péninsule est le deuxième partenaire commercial de la Fédération russe, après l'Allemagne. Les contacts et les visites entre Silvio Berlusconi et le Maître du Kremlin sont chaleureux et étroits à tel point que l'actuel président du Conseil des Ministres italien parle de Vladimir Poutine comme de "son ami ». Leur amitié ${ }^{1}$ est mise en relief par les presses italienne et russe dès les titres des articles qui les concernent.

2 Le site du Ministère des affaires étrangères italien qualifie ces relations de "privilégiées $»^{2}$. Au cours des dernières années, la politique étrangère italienne a toujours soutenu le rapprochement entre la Russie et l'Occident au sein de l'Union Européenne, de l'OTAN, de l'OMC et de l'OCDE. La péninsule accroît son rayonnement international ${ }^{3}$ et sert de « pont » entre l'Occident et la Russie. La force de cette relation bilatérale se vérifie à travers la très grande fréquence des contacts au plus haut niveau.

3 Or, en dépit de la fréquentation si intense et des liens si étroits et si riches que la Russie entretient avec l'Italie, l'image de son ancien Président et actuel premier Ministre, qui s'est tant investi dans les relations italo-russes, n'est pas positive dans la presse italienne tout comme dans le reste de la presse occidentale. On parle de lui comme d'un personnage froid, mystérieux, autoritaire, qui bafoue les droits de l'homme. Les appellations que les journalistes italiens utilisent pour l'identifier sont évocatrices de cette image. Elles sont très nombreuses : ex agente del KGB (ancien agent du KGB), ex ufficiale del KGB (ancien officier du KGB), l'agente del Cremlino (l'agent du Kremlin) comme si l'on voulait signifier qu'il n'a jamais quitté ses anciennes fonctions, il capo del Cremlino (le chef du Kremlin), il leader del Cremlino (le leader du Kremlin), il padrone del Cremlino (le maitre du Kremlin), lo zar di tutte le Russie (le tsar de toutes les Russies). 
Il serait certes très intéressant d'analyser les modalités et les raisons de la construction de cette image au fil du temps, mais notre propos se limite à analyser les distorsions éventuelles provoquées par de tels présupposés. Comment le lecteur italien a-t-il pris connaissance de la conférence de presse tenue par Vladimir Poutine le $1^{\mathrm{er}}$ juin 2007 à la veille du G8 qui s'est déroulé en Allemagne du 6 au 8 juin ?

(Lire le Texte de cadrage)

\section{Contexte international}

5 Il nous semble utile de rappeler que le climat international de l'époque est tendu. Pendant les premiers mois de l'année 2007, une série de déclarations faites par le gouvernement américain troublent la situation internationale, notamment le discours du 7 février au sénat de R. Gates, secrétaire de la Défense et la publication du Strategic Plan 2007-2012 de la part du Département d'État américain. Le secrétaire de la défense affirme qu'il est nécessaire d'augmenter le budget pour les armements car la situation politique interne russe n'est pas bien définie et que cet état de choses représente une menace pour la sécurité des USA. Le Strategic Plan 2007-2012 est en outre plus sévère que jamais envers la Russie: il souligne surtout les failles de la démocratie russe, et notamment les difficultés rencontrées par la presse russe ${ }^{4}$. Poutine répond à ces propos, le 10 février 2007 lors de la $43^{\text {e }}$ conférence sur la politique de sécurité à Munich. Lors de son discours, le président russe dénonce l'unilatéralisme des États-Unis, critique l'élargissement de l'Alliance atlantique à des pays qui faisaient jadis partie de l'URSS. Le projet américain de bouclier antimissile est jugé intolérable. L'attitude américaine à travers le monde alimente une course aux armements ${ }^{5}$.

Dans ce contexte, le $1^{\text {er }}$ juin 2007, Vladimir Poutine convie, dans sa résidence d'été de Novo-Ogaryovo, les huit journalistes représentant les pays membres à un dîner au cours duquel il donne une conférence de presse. Pour l'Italie, le journal invité a été le Corriere Della Sera ${ }^{6}$ representé par Franco Venturini ${ }^{7}$, premier éditorialiste du journal pour la politique internationale, et par Fabrizio Dragosei, correspondant permanent pour le $C D S^{8}$ à Moscou. Les deux journalistes parlent couramment russe.

\section{Corpus et méthodologie}

7 Notre hypothèse de travail est que les rédacteurs de l'article proposé aux lecteurs italiens cherchent à dramatiser les propos du président russe, à créer l'événement, à «raconter une histoire » qui correspondrait à l'image et au discours attendus de Poutine. Le discours rapporté des rédacteurs semble construit d'après l'image de Poutine dans la presse occidentale.

Notre approche sera dénotative, et portera donc sur le contenu de l'article du Corriere et elle sera également connotative. Il convient d'analyser les éléments constitutifs du document proposé au lecteur italien : le titre, les sous-titres, le chapeau, les questions et les réponses. L'organisation du texte renvoie à l'univers de l'argumentation car le choix des prémisses influence la réception. Notre objectif est de dégager les choix des journalistes tout en étant conscient que les limites d'un article ne permettent pas de mettre en relief toutes les nuances. 
Une conférence de presse semble impliquer que l'intervention du rédacteur sur l'information est minimale. Le journaliste est censé rapporter le discours qu'il a entendu. La partie destinée au commentaire est moindre que dans un éditorial, une tribune ou un dossier. La présence des autres journalistes des pays du G8 devrait représenter une "garantie" supplémentaire de traitement objectif de l'information. Un lecteur avisé et polyglotte peut vérifier ce qui a été dit à Novo-Ogaryovo, et, pourquoi pas, confronter les différentes productions des journalistes présents.

Quelles stratégies mettent en œuvre F. Venturini et F. Dragosei pour remplir leur contrat médiatique? La conférence de presse s'apparente beaucoup à une interview. Toutefois, il n'y a pas que trois instances (intervieweur, interviewé et un tiers absent), il y en a bien plus. Les intervieweurs cherchent des vérités ${ }^{9}$, essayent de faire découvrir au public absent des éléments nouveaux pour qu'il puisse, ensuite, se faire une opinion tandis que l'interviewé cherche à communiquer et à faire connaître des éléments d'information qui sont, pour lui, fondamentaux.

Rappelons que huit journaux sont impliqués : l'hebdomadaire allemand Der Spiegel, le quotidien économique américain Wall street Journal, le quotidien économique japonais Nikkey, le quotidien britannique The Times, le quotidien russe Kommersant, le quotidien italien Corriere della Sera, le quotidien français Le Figaro et The Globe and Mail, quotidien canadien de langue anglaise ${ }^{10}$. La triangulation est à multiplier par huit dans notre cas. Aussi les journalistes du CDS ont-ils pu entendre non seulement les réponses à leurs questions mais également celles des autres journalistes.

11 Comment les journalistes italiens ont-ils profité de cette situation qui leur permettait de « voir Vladimir Poutine » également à travers les « lunettes» de plusieurs cultures différentes? Transmettent-ils à leurs destinataires «absents » cette richesse ? Leur transmission est-elle fidèle? Exhaustive? Partielle? Quelles sont les raisons de leurs choix?

Rien de tel que de confronter le texte source de la conférence et l'article dont a disposé le lecteur italien ${ }^{11}$. La fédération de Russie accorde une grande importance à la communication. Les conférences de presse du Président sont traduites en anglais par le Kremlin et mises en ligne en russe et en anglais ${ }^{12}$. Ceci constitue la procédure habituelle, mais le texte mis en ligne en juin 2007 n'est plus disponible dans les archives du web proposées au public par le Kremlin. Aucune explication n'a été donnée pour cette absence ou censure déguisée.

\section{Présentation pour le lecteur du Corriere della Sera}

12 Il convient de commencer notre analyse par les éléments que la rédaction du CDS a sélectionnés pour introduire le sujet. Cette partie est fondamentale dans un journal. Un titre est lu en moyenne cinq fois plus que le corps du texte ${ }^{13}$. Le titre opère comme un panneau de signalisation, donne du sens à ce qu'on va lire par la suite. Le titre annonce la couleur. Il engage l'auteur à tenir sa promesse ${ }^{14}$.

Quels sont ces éléments dans l'article du CDS?

Les éléments dans notre cas sont nombreux: surtitre, titre, sous-titre, photo et chapeau. Ceux-ci vont créer la mise en scène que les journalistes italiens ont choisie pour capter l'attention du lecteur et l'informer sur l'événement. Charaudeau compare le travail du journaliste à celui du metteur en scène. Comme ce dernier, il a une matière 
bien déterminée à transmettre, mais il est libre de la présenter de plusieurs façons ${ }^{15}$. Examinons les choix de mise en scène. Et commençons par le surtitre, dont le but est de mettre en situation le lecteur:

Tensione con gli Usa su armamenti, diritti umani, caso Litvinenko. «Non uso un linguaggio

da luna di miele ${ }^{16}$ ».

Les faits sont annoncés. On n'utilise pas d'articles, ni d'adjectifs pour modeler le réel, mais seulement une suite de noms. L'effet attendu est l'objectivisation de la réalité par le dépouillement linguistique. Le deuxième élément du surtitre "droits de l'homme " n'est pas spécifié davantage (Où ?) mais sa collocation le rend menaçant. Il se trouve entre «tension avec les Usa » et "cas Litvinenko » qui a fait tant parler la presse transalpine à cause des implications des services secrets italiens. Le lecteur est déjà plongé dans un univers inquiétant. Enfin, on rapporte une phrase hors contexte de Poutine «Je n'utilise pas un langage de lune de miel ». L'absence de contexte de cette phrase sert à construire l'image menaçante de Poutine déjà avant même la lecture du titre.L'auteur de la phrase proposée reste inconnu bien que les indices donnés ne laissent pas de place au doute. Le suspens n'est qu'apparent. Les prémisses sont données.

Le titre :

Putin: pronto a puntare i missili sull'Europa

Ici la mise en scène se précise davantage. Le responsable est nommé. L'utilisation des deux points après le nom peut faire penser en italien qu'il s'agit d'une déclaration du président russe bien que normalement les guillemets soient plus couramment utilisés pour indiquer le discours direct. Le tableau est de plus en plus inquiétant. La menace devient réelle et parlante. Le lecteur est obligé de se sentir impliqué par le titre: Poutine est prêt à braquer ses missiles sur l'Europe. L'un des critères fondamentaux pour retenir l'attention des lecteurs est la proximité. Qu'est-ce qui peut être plus angoissant que de savoir que les missiles russes sont braqués sur notre continent ?

Le sous-titre :

Intervista al presidente russo: le manifestazioni represse? Sciocchezze

Le lecteur est désormais certain que les menaces ont été prononcées par Poutine en personne. Les journalistes ajoutent une information supplémentaire sur le texte qui va suivre : l'article porte sur une interview au président russe. Les journalistes n'évoquent nulle part dans le titre le fait qu'il s'agit en réalité d'une conférence de presse. Exercice qui demande bien plus d'ouverture et de disponibilité qu'une interview du fait que l'interviewé est soumis aux questions de plusieurs instances médiatiques.

De nouveau, le discours redevient direct. Par conséquent les affirmations affichées prennent un relief plus important. Et que dit le président russe? Les manifestations réprimées? Bagatelles. Il s'agit de la première allusion à la situation interne du pays. La phrase est elliptique et privée du contexte où elle a été utilisée. Hors contexte, elle se prête à au moins deux interprétations, toutes les deux négatives :

a. Poutine affirme qu'il n'y a jamais eu de manifestations réprimées en Russie. Toutefois la presse italienne en avait beaucoup parlé et avait également critiqué l'attitude du gouvernement russe à ce sujet. Désormais c'est une opinion commune partagée par la presse et les lecteurs. Ainsi, ce sous-titre équivaut à dire que Poutine est un menteur. Et cela d'entrée de jeu...

b. Les manifestations étaient peu importantes, rien de grave ne s'est vraiment passé.

Il est difficile de partager cette lecture de la réalité pour le lecteur du CDS. Ne pas 
prendre en considération les manifestants (dont la presse italienne avait parlé), les ignorer est une attitude très peu démocratique. Ce jugement de valeur est commun au lecteur et aux rédacteurs. Dans ce cas, la prémisse sous-jacente est : Poutine est un dictateur. Le choix pour le lecteur est de considérer Poutine soit comme un menteur soit comme un dictateur. événementiel. Il s'agit d'un portrait en couleurs et de petit format. La source de l'image est la principale agence de presse italienne (ANSA) comme l'annonce la légende qui accompagne la photo et qui sert à identifier le personnage. La photo n'est pas datée. Par ailleurs, il a été impossible de savoir s'il s'agit d'une photo d'archives ou d'une photo prise lors de la conférence de presse dont il est question comme les deux microphones noirs au centre de la photo semblent le suggérer.

«L'image fixe semble plus réelle, plus crédible que le cinéma, que l'actualité télévisée, alors que sa charge idéologique n'est pas moins faible, bien au contraire, puisque, justement, on s'en méfie moins » affirme Marc Arabyan ${ }^{17}$. Que dit donc cette image ? Quel est son sens?

allons ainsi analyser le montage, l'angle de vue, la composition, le décor de cette photo et ensuite nous allons nous intéresser à l'expression corporelle du personnage mis en scène. Ces éléments nous permettront de voir si ces choix correspondent à un point de vue spécifique des rédacteurs du CDS.

a plan américain où la tête touche le bord supérieur de l'image et où le corps est coupé à peu près au niveau de la ceinture. Poutine est penché en avant, il semble prendre appui sur un bureau, exprimant le poids de la charge, la tension de la situation, mais aussi la volonté d'agir. Il est légèrement tourné vers la droite, un stéréotype de l'homme qui se prépare à agir. Le leader russe est photographié en très légère plongée, du haut vers le bas. Cet angle de vue, du point de vue du personnage sous les projecteurs, suggère la contrainte, la situation difficile qu'il doit affronter. Sur la photo, Poutine est montré presque de face sur un alignement vertical : front, nez, microphone. L'image est de type statique, le résultat est une image équilibrée, ce qui contribue à donner plus de stabilité et fermeté au personnage représenté.

24 Pour ce qui est du décor, il est presque inexistant car le cadrage est très serré. Le seul élément identifiable est le dossier du fauteuil sur lequel Poutine est assis. Il s'agit d'un fauteuil doré qui évoque, par sa forme et ses éléments de décoration, presque le trône d'un souverain. Le président russe est habillé de façon très élégante : costume sombre, chemise blanche, cravate. Tout cela suggère que nous sommes en présence d'un homme de pouvoir.

Mais ce qui attire davantage l'attention du lecteur de cette image est l'expression du visage et du regard de Poutine: le visage occupe presque un tiers de la photo. L'expression est celle de la concentration et de la tension suggérées par le plissement $\mathrm{du}$ front. Le regard corrobore cette impression de concentration car ses yeux clairs semblent fixer très attentivement un interlocuteur que le lecteur ne voit pas. Le regard est froid et va dans le même sens que le corps et que l'angle de vue, c'est-à-dire vers le haut. Poutine n'est pas représenté en train de parler et de communiquer. Sur cette image, il écoute et regarde. Les traits de son visage sont tirés et creusés. Pour ce qui est de la position des mains, elles ne sont pas visibles. Toutefois, la posture du président russe et les manchettes de la chemise nous permettent de comprendre que les mains 
sont immobiles, qu'elles reposent sur le bureau. Il s'agit d'un signe ultérieur de concentration, de stabilité et de fermeté.

Ainsi, tout dans cette image semble suggérer au lecteur que le président russe est très attentif à ce qui se passe, qu'il est inquiet et qu'homme de pouvoir est prêt à agir. La photo analysée semble renforcer le message véhiculé par le titre choisi par les journalistes du CDS. La photo est un moyen très puissant de rapprocher l'information du lecteur et de la rendre réelle. La menace évoquée dans le titre est ainsi personnifiée grâce à l'image de Poutine que les journalistes ont choisie.

Et que se passe-t-il dans le chapeau? Le chapeau joue un rôle fondamental dans un article de presse : il permet au lecteur de connaitre l'essentiel de ce qu'il va lire. Dans les guides de rédaction pour journalistes, on affirme que « les éléments d'informations $\mathrm{du}$ titre et $\mathrm{du}$ chapeau doivent tous se retrouver dans le texte $»^{18}$. Le chapeau est une incitation à la lecture.

Et d'après les règles du journalisme, il doit être possible de comprendre un article sans lire le titre et le chapeau, et inversement. Est-ce vrai dans le cas qui nous occupe ? Et si telles sont les règles, cela signifie que la clé de la conférence, les informations fondamentales sont données dans cette présentation. Quelles sont donc les informations principales d'après nos rédacteurs? Je cite :

NOVO-OGARYOVO (Russia) - Sono passate le otto di sera, Vladimir Putin è in ritardo perché $\grave{e}$ andato a visitare la vedova di Eltsin. I rappresentanti dei giornali invitati dal Cremlino, uno per ogni Paese del G8 che si apre mercoledì in Germania, lo attendono nella dacia presidenziale di Novo-Ogaryovo, immersa in un bosco stupendo oltre il quale spuntano come funghi le seconde case dei nuovi miliardari moscoviti. L'atmosfera è rilassata, funzionari e guardie del corpo giocano a biliardo per ingannare il tempo. Ma quando il Presidente arriva e comincia un incontro che poco dopo si trasferirà a tavola, l'amichevole informalità che regna nella dacia diventa poca cosa davanti alla rudezza delle parole. Sì, risponde Putin a una nostra domanda, $i$ missili nucleari russi torneranno a essere puntati contro città $e$ obiettivi militari europei se gli Usa insisteranno a modificare l'equilibrio strategico con il coinvolgimento di Polonia e Repubblica Ceca nella creazione di uno «scudo » anti- balistico. Il messaggio è forte, ma conviene cominciare dall'antipasto ${ }^{19}$.

Le lecteur a l'impression de ne plus lire un article censé l'informer sur le contenu d'une interview menaçante du président russe comme le sous-titre l'annonce. Les rédacteurs présentent l'information à la manière d'un récit, ou d'un thriller où l'on décrit la scène du crime en fournissant une multitude de détails pour que le lecteur plonge dans l'ambiance et soit davantage surpris lorsque la menace apparait. Ici, l'heure est donnée (20 heures), le président russe se fait attendre par les journalistes du G8 qu'il a invités. Il est en retard car il est allé voir la veuve d'Eltsine. Le lecteur est également mis au courant $\mathrm{du}$ fait que la rencontre avec la presse se déroulera dans la datcha présidentielle de Nova-Ogaryovo, située au milieu d'un bois magnifique où se multiplient les résidences secondaires des nouveaux riches de Moscou. On nous décrit même les occupations des gardes du corps (ils jouent au billard) pour tuer le temps. L'ambiance est détendue, mais dès que le Président arrive et que la rencontre commence, le climat amical disparaît. Les mots utilisés par Poutine sont très durs car il dit que les missiles russes seront de nouveau braqués sur les villes et sur les objectifs militaires européens si les Américains continuent de modifier l'équilibre stratégique en entraînant la Pologne et la République Tchèque dans la créationd'un "bouclier " antimissile. 
Quel est l'essentiel de ce passage? La menace de Poutine de braquer ses missiles sur l'Europe si les USA persévèrent avec le bouclier antimissile.

Et alors quel est le sens de tous les autres détails? Les détails illustrés ne peuvent servir qu'à construire l'image du président que le journaliste propose. Le président se fait attendre, ce qui pourrait signifier implicitement, qu'il ne respecte pas les règles de la société civile. Tout est beau et détendu jusqu'à son arrivée ou plus précisément jusqu'à ce qu'il parle. Il s'agit donc d'un être menaçant et inquiétant.

32 La lecture du texte source nous fournit des informations supplémentaires qui nous éclairent sur le déroulement de cette soirée à Nova-Ogaryovo : Poutine s'est rendu à la cérémonie des 40 jours après la mort d'Eltsine. Il s'agit d'une cérémonie très importante en Russie. Son retard est justifié. Nous apprenons également que Vladimir Poutine a organisé pour la conférence de presse un banquet où chaque plat faisait honneur à l'un des pays du G8. En outre, le président Poutine a prononcé un discours de bienvenue pour accueillir ses invités. Il s'agit d'un discours relativement long (environ 25 lignes et 356 mots) dans lequel Poutine souligne le rôle clé que jouent les pays du G8 dans l'économie mondiale. Il met également en avant que lors du G8 en Allemagne les pays membres discuteront de thèmes internationaux importants pour la communauté internationale comme les Balkans. Il affirme clairement sa confiance dans l'utilité d'une discussion ouverte et honnête sur tous les problèmes internationaux même s'il $s^{\prime}$ agit de problèmes difficiles à résoudre ${ }^{20}$. Et il conclut son discours en remerciant les journalistes pour l'intérêt qu'ils portent au travail de la Russie. Il déclare ouvertement sa disponibilité à expliquer dans le détail la position de la Russie sur les questions susceptibles d'intéresser l'opinion publique ${ }^{21}$.

3 Le banquet organisé en l'honneur de la presse internationale ainsi que le discours introductif peuvent être considérés comme les éléments mis en scène par le Président russe pour proposer, construire son image auprès de l'opinion publique. Le quotidien italien les efface d'un coup de plume.

Aucun élément d'information qui pourrait servir une image moins négative du Président russe n'est fourni au lecteur. La description proposée dans le chapeau est dramatisante, les rédacteurs cherchent à effrayer le lecteur ou tout au moins à provoquer chez lui des instincts de peur. La mise en scène du CDS se termine par le commentaire du journaliste et par son invitation à la lecture de la conférence de presse. Le journaliste tire en effet sa conclusion et la présente comme une donnée objective. Le message est fort dit-il. Et il prévient: mais il convient de commencer par le commencement. Ce sont les mots de la fin du chapeau. Ils sonnent comme une menace sur le contenu de la conférence de presse que le lecteur s'apprête à lire. La couleur est clairement annoncée.

\section{Conférence de presse}

L'analyse quantitative montre immédiatement que le texte source a été considérablement réduit. Rien d'étonnant vu le nombre important de questions et la longueur des réponses du Président Poutine. 80,5 \% du texte source en anglais (19 181 mots) est consacré au discours de Poutine (15437 mots) tandis qu'en italien ce pourcentage n'est que de $71,9 \%$ du texte source italien (1 897 mots). Ainsi, le texte proposé au lecteur italien correspond à $9,9 \%$ de la conférence. 
Certes, les journalistes ne pouvaient pas rapporter l'intégralité de la conférence à cause des contraintes d'espace imposées par la nature même du quotidien; mais quels ont été leurs choix? Les journalistes ont choisi de diminuer de façon significative (presque $10 \%)$ la part de parole du « locuteur auteur » du discours rapporté. Cette réduction est d'autant plus significative que l'anglais est une langue bien plus synthétique que l'italien. Or la conférence de presse a été organisée à l'initiative de Poutine qui souhaitait donc être entendu et faire connaître son point de vue à l'opinion publique mondiale, mais son «temps de parole » pour le récepteur italien a été réduit au profit $\mathrm{du}$ « temps de parole » des journalistes.

37 La conférence de presse est très "riche ", environ 43 questions, certaines étant le regroupement de plusieurs. L'interview du CDS ne compte que 19 questions. Les choix des sujets présentés répondent essentiellement aux critères de la proximité. Tout ce qui est susceptible d'intéresser le lecteur italien et surtout susceptible d'avoir des retombées sur sa vie quotidienne est traité. Par conséquent, les rédacteurs italiens ont mis de côté toutes les questions posées par le quotidien Nikkei qui portaient essentiellement sur la politique asiatique de la Russie et sur les relations Japon/ Russie, les questions concernant les relations entre la Russie et l'Allemagne et pour finir l'interdiction pour les Russes d'exporter des produits biologiques humains dans le cadre de la recherche médicale.

38 Ainsi, dans l'ordre de présentation pour le lecteur italien, les sujets traités par le CDS sont : la fin de l'amitié entre la Russie et l'Occident et le retour à la guerre froide ; l'installation $\mathrm{du}$ bouclier antimissiles en Pologne et en République Tchèque; l'éventuelle riposte russe avec la construction de bases à Cuba et au Venezuela; les nouvelles cibles en Europe si le projet du bouclier se poursuit ; le traité INF en danger et les considérations sur le traité CFE; les réactions de la Russie à une éventuelle entrée de l'Ukraine au sein de l'OTAN et de l'Union Européenne ; la détérioration des relations entre l'Otan et la Russie; les divergences au sujet du nucléaire avec l'Iran; le fait que l'Iran ne doit pas posséder l'arme nucléaire; le Kosovo ; l'éventuelle exclusion de la Russie du G8 à cause des défauts de sa démocratie; les manifestations réprimées en Russie; la grande popularité de Poutine en Russie et son contrôle sur les médias; l'extradition d'Andrej Lugovoj pour l'affaire Litvinenko ; la fin du mandat de Poutine et ses projets politiques pour les années à venir; l'opinion de l'épouse de Poutine sur ses projets politiques; le fait que Shell et BP perdent leurs licences d'exploitation en Russie ; Aeroflot et Alitalia ; le président Sarkozy ami des USA et fervent défenseur des droits de l'homme ${ }^{22}$. Les questions ne sont pas identifiées : le lecteur ne connait pas non plus les noms des journalistes conviés par Poutine, ni ceux des journaux qu'ils représentent.

Une fois illustrés les choix de sujets, il nous semble utile aux fins de l'analyse de constater ce qui se passe à l'intérieur des questions retenues par le quotidien italien. Quels sont les changements opérés dans le message proposé au lecteur? Nous ne citerons que quelques exemples significatifs. Commençons par la première question de la conférence de presse. Elle a été posée par l'hebdomadaire allemand «Der Spiegel », et choisie par le CDS pour commencer son article. Il s'agit de la fin de l'amitié entre l'Occident et la Russie, et du début d'une nouvelle guerre froide. C'est la question qui donne le ton au texte italien. 
Texte source : M. President, it seems like Russia is not very fond of the West. Our relations have somewhat deteriorated. And we can also mention the deterioration of your relations with America. Are we once again approaching a Cold War?

Texte CDS : Signor Presidente, non c'è più amore tra Russia e Occidente, lei parla di imperialismo Usa come si faceva ai tempi dell'Urss; siamo già in un clima da nuova guerra fredda?

Cette question compte 38 mots en texte source et 30 dans le texte italien. Le rédacteur semble se limiter à synthétiser la question de son confrère allemand. Or, son intervention va bien plus loin qu'un raccourci. Le journaliste italien n'a pas simplement traduit et synthétisé. Il a ajouté deux mots clés (impérialisme USA) pour étoffer le scénario "guerre froide». Cet ajout ne semble pas innocent dans le contexte international du moment. La façon de transcrire la réponse confirme la volonté de « donner une interprétation » aux mots prononcés.

Texte source: VLADIMIR PUTIN: One can hardly use the same terminology in international relations, in relations between countries, that would apply to relationships between people-especially during their honeymoon or as they prepare to go to the Civil Registry Office.

Throughout history, interests have always been the main organising principle for relations between states and on the international arena. And the more civilised these relations become, the clearer it is that one's own interests must be balanced against the interests of other countries. And one must be able to find compromises to resolve the most difficult problems and issues. One of the major difficulties today is that certain members of the international community are absolutely convinced that their opinion is the correct one. And of course this is hardly conducive to creating the trusting atmosphere that I believe is crucial for finding more than simply mutually acceptable solutions, for finding optimal solutions. However, we also think that we should not dramatise anything unduly. If we express our opinions openly, honestly and forthrightly, then this does not imply that we are looking for confrontation. Moreover, I am deeply convinced that if we were able to reinstate honest discussion and the capacity to find compromises in the international arena then everyone would benefit ${ }^{23}$. And I am convinced that certain crises that face the international community today would not exist and would not have had such a dire impact on the internal political situation in certain countries. For example, events in Iraq would not be such a headache for the United States. This is the most vivid, sharpest example but, nevertheless, I want you to understand $\mathrm{me}^{24}$. And as you recall, we were opposed to military action in Iraq. We now consider that had we confronted the problems that faced us at the time with other means then the result would have been - in my opinion - still better than what we have today. It is for that reason that we do not want confrontation; we want to engage in dialogue. However, we want a dialogue that acknowledges the equality of both parties' interests ${ }^{25}$.

Texte CDS «Nelle relazioni internazionali non si usa un linguaggio da luna di miele. Vanno sempre difesi i propri interessi nazionali, e la coesistenza consiste nel farlo insieme, in uno spirito di compromesso. Qualcuno nella comunità internazionale crede che le sue idee e i suoi interessi siano valori assoluti da affermare con ogni mezzo. Questo non aiuta. Faccio un esempio: se avesse prevalso lo spirito di compromesso, $i$ nostri consigli sarebbero stati ascoltati e gli Usa non avrebbero attaccato l'Iraq. Certo oggi la situazione sarebbe migliore ma non voglio nemmeno drammatizzare i contrasti, non è proprio il caso di parlare di guerra fredda ${ }^{26} »$.

41 Que s'est-il passé ? La réponse de Poutine dans le texte source est très développée. Elle compte 348 mots. La réponse italienne 101. Théoriquement, ce rapport d'1 à 3 semble acceptable, la concision étant l'une des qualités demandées aux journalistes. Il faut distinguer l'essentiel de l'accessoire ${ }^{27}$. Mais peut-on dire que Poutine exprime à plusieurs reprises dans ce passage le souhait d'établir un dialogue honnête et équitable avec ses partenaires et qu'il ne cherche pas l'affrontement? Le journaliste italien préfère ne pas évoquer ce souhait de Poutine, mais il utilise en revanche le terme 
" guerre froide », gravement connoté. Il s'agit de frapper le lecteur. Il est important de souligner que Poutine n'utilise jamais le terme " guerre froide » dans la conférence.

La $2^{\mathrm{e}}$ réponse est également significative du point de vue du nombre de mots utilisés dans la transcription en italien. Il s'agit de la réponse à la question posée par le Wall Street Journal sur le bouclier antimissile que les Américains voudraient installer en Pologne et en République tchèque. Cette question compte 119 mots $^{28}$. La réponse de Poutine est très longue. Il s'agit d'un véritable discours, complexe et cohérent. Poutine veut expliquer le point de vue de la Fédération de Russie face à l'initiative américaine. Les enjeux sont donc de taille : la question dans le texte source compte 985 mots. Dans le texte italien 219. À eux seuls, les chiffres expriment clairement les choix des journalistes. Le rapport est ici presque d' 1 à 5 . Et pourtant, il s'agit du sujet le plus intéressant pour la presse occidentale: pour la première fois dans l'histoire, dit le président russe, des éléments du système nucléaire américain sont présents sur le continent européen. Poutine souhaite attirer l'attention de tout le monde sur cette réalité. Lors de la conférence, il s'adresse même aux lecteurs des journalistes invités. Tous doivent entendre son discours.

Texte source : I draw your attention and that of your readers to the fact that, for the first time in history - and I want to emphasize this - there are elements of the U.S. nuclear capability on the European continent ${ }^{29}$.

Et que se passe-t-il ? Tous les efforts rhétoriques de Poutine pour illustrer l'attitude et la perception de la Russie face à cette initiative américaine disparaissent ou presque. Poutine redit ici que les Russes souhaitent avant tout être entendus. Ils souhaitent que leur position soit comprise.

Texte source : (...) And now I would like to give a definite answer to your question: what do we want? First of all, we want to be heard. We want our position to be understood.

Et il ajoute également que les Russes n'excluent pas que leurs partenaires américains reconsidèrent leur décision :

Texte source : We do not exclude that our American partners might reconsider their decision.

Les Russes n'imposent rien à personne. Comme il le dit clairement :

Texte source : We are not imposing anything on anyone.

Mais les Russes agissent comme des gens de bon sens et ils s'attendent à ce que tous les autres agissent de la même façon.

Texte source: But we are proceeding from common sense and think that everyone else could also use their common sense (...).

Poutine affirme que l'initiative américaine est en train de détruire l'équilibre stratégique mondial et que pour le rétablir sans avoir recours à un système de défense antimissiles, les Russes vont être obligés de créer un système pour se préserver de la défense antimissile, et qu'ils sont maintenant en train de le faire.

Texte source : It is destroying the strategic equilibrium in the world. In order to restore that balance without setting up a missile defence system we will have to create a system to overcome missile defence, and this is what we are doing now (...).

C'est une nécessité pour les Russes de garantir leur sécurité (We need to ensure our security.)

Le président conclut en s'interrogeant sur les raisons de cette obstination américaine d'installer un bouclier antimissile qui n'a, à son avis, aucune raison d'exister. Il avance alors l'hypothèse que peut-être les Américains veulent pousser les Russes à engager des 
actions de représailles dans le but d'empêcher un rapprochement plus étroit entre la Russie et l'Europe. Et, si tel était le cas (selon lui il ne s'agit que d'une possibilité et non d'une certitude), il y a erreur car ce n'est pas ainsi qu'on améliore la paix et la sécurité internationales.

Texte source : But I sometimes think to myself: why are they doing all this? Why are our American partners trying so obstinately to deploy a missile defence system in Europe whenand this is perfectly obvious - it is not needed to defend against Iranian or - even more obvious - North Korean missiles? (We all know where North Korea is and the kind of range these missiles would need to have to be able to reach Europe.) So it is clearly not against them and it is clearly not against us because it is obvious to everyone that Russia is not preparing to attack anybody. Then why? Is it perhaps to ensure that we carry out these retaliatory measures? And to prevent a further rapprochement between Russian and Europe? If this is the case (and I am not claiming so, but it is a possibility), then I believe that this would be yet another mistake because that is not the way to improve international peace and security.

Ces éléments informatifs, que nous avons voulu détailler en raison de leur importance, sont totalement absents dans la transcription du CDS. Les journalistes n'ont pas cru utile de fournir au lecteur italien ce qui pouvait éclairer la position de Poutine. De plus, comme dans le premier exemple, ils dramatisent et présentent un scénario catastrophe: ils disent que les Russes ont l'intention de rééquilibrer les moyens défensifs par des instruments offensifs plus efficaces.

Texte CDS: Intendiamo invece riequilibrare gli strumenti difensivi con più efficaci strumenti offensivi, senza tuttavia aumentare le spese militari, ma sappiamo che questo rischia di riaprire una corsa agli armamenti di cui non saremo comunque responsabili.

51 Encore une fois, le souhait de Poutine d'être entendu n'est pas satisfait.

Et pour conclure sur ce choix d'exemples d'analyse quantitative et du contenu, prenons en considération une réponse très courte. Les modifications et les éléments censurés sont ici encore plus facilement visibles. Il s'agit de la troisième réponse. La question a été posée par Der Spiegel. Le sujet est très inquiétant : l'éventuelle riposte des Russes au bouclier des États-Unis. Il est utile pour comprendre l'organisation de la réponse de nous attarder d'abord sur la forme de la question. Dans le texte source, la question ne compte que 29 mots et est très incisive. Le journaliste allemand demande si les Russes seraient disposés à considérer la possibilité d'installer un système de défense antimissile russe dans des zones proches des États-Unis, par exemple à Cuba.

Texte source: A short additional question: would you be prepared to consider the possibility of deploying a similar, Russian missile defence system somewhere near the United States, for example in Cuba?

52 Le journaliste de l'hebdomadaire allemand prend toutes les précautions d'usage. Il présente la riposte russe comme une possibilité. Il parle explicitement de système de défense. Que se passe-t-il dans le texte italien? Le journaliste du CDS propose à son lecteur potentiel la transcription suivante qui compte 20 mots et dont le sens est bien différent de la question véritable.

Texte CDS : Le viene mai la tentazione di restituire pan per focaccia all'America, di

collocare missili russi a Cuba o in Venezuela? ${ }^{30}$

53 Ici le CDS suggère une tentation possible, sûrement négative dans ce contexte car elle présuppose que Poutine pourrait prendre des mesures militaires lourdes. Il succomberait à la tentation de la vengeance, de la loi du talion. Nous sommes en plein scénario catastrophe. Et en plus de Cuba, il y a le Venezuela, sans doute pour dramatiser encore plus en évoquant l'un des pays où les États-Unis ont des intérêts 
pétroliers considérables. Cette perspective crée un danger supplémentaire pour affoler le destinataire de ce message. Les modalités de construction de la réponse sont tout aussi éclairantes sur le parti pris du CDS. Dans le texte source, la réponse compte 125 mots. Dans le CDS, 24 mots. Le rapport est ici d'1 à 5. d'éléments présents dans le discours de Poutine sont écartés dans la retranscription proposée. Il s'agit d'éléments qui auraient nuancé l'image négative de Poutine en y ajoutant d'autres facettes. De façon systématique, les journalistes ont, par leurs omissions et par la construction de l'article, réduit à zéro tous les efforts du Président russe pour renouveler son image auprès de l'opinion publique occidentale. En effet, dans le texte source, Poutine se présente d'une manière qui ne transparaît pas du tout dans le discours rapporté du CDS. Il parle clair et net, il ne cherche pas l'affrontement, il souhaite être compris, il souhaite un dialogue équitable, il subit les initiatives américaines, il respecte les règles du droit international, il se soucie des intérêts des autres, il cherche un compromis, il s'oppose à la course aux armements, il ne veut pas être obligé de prendre des initiatives militaires pour se protéger, il est disposé à croire que les Américains peuvent changer d'avis, il souligne, preuves à l'appui, que la Russie a 
beaucoup changé, il affirme être un "pur démocrate ", il engage des politiques pour lutter contre la pauvreté, il reconnaît qu'il peut se tromper, il affirme ne pas contrôler les médias, il reconnaît aux citoyens le droit de manifester (dans des cadres bien définis). Et enfin, le texte source nous dévoile un homme plein d'humour qui rit souvent pendant la conférence de presse. Ces différentes facettes de la personnalité de Poutine s'opposent à l'image manichéenne qui ressort du texte du CDS, dès le titre. N'est-on pas en droit de dire que l'instance médiatique a manipulé l'information? Il faudrait probablement nuancer ce propos : les journalistes ne peuvent pas tout dire, ils sont obligés d'opérer une sélection, de choisir les éléments d'information à proposer au lecteur. Ils doivent être à la fois crédibles et compréhensibles.

Or, pour se mettre à la portée de leur lectorat ils sont parfois conduits à simplifier leur discours, à en grossir les traits pour frapper. La conférence de presse de Poutine constitue un véritable défi pour les journalistes. La situation de communication est extrêmement complexe : dans notre analyse, nous avons parlé d'une «triangulation à huit " avec 8 journaux, de huit pays différents. Les relations internationales sont tendues. La durée de la conférence ajoute à la difficulté. Comment réduire une telle complexité en quelques colonnes dans un journal ? Et dans un délai très court ? Quelles ont été les priorités des journalistes dans ce cas? La priorité semble avoir été de faire croire aux lecteurs que la situation est difficile, que les enjeux pour la securité internationale sont importants et qu'il faut se méfier de Poutine.

Franco Venturini et Fabrizio Dragosei ont donc noirci le tableau et ils l'ont simplifié. Dans ce sens, ils ont bien rempli leur contrat.

\section{BIBLIOGRAPHIE}

Arabyan M., Lire l'image, Paris, L'Harmattan, 2000.

Cevasco F., De Stefano D. (dir.), Come si scrive il Corriere della Sera, Milano, BUR, 2003.

Champagne P., 1991, La construction médiatique des " malaises sociaux", Actes de la recherche en Sciences sociales, $\mathrm{n}^{\circ}$ 90, p. 64-75, 2003.

Charaudeau P., Le discours politique, Cahors, Vuibert, 2005.

Charaudeau P., Les médias et l'information. L'impossible transparence du discours, Bruxelles, De Boeck, 2005.

Durandin G., L'information, la désinformation et la réalité, Paris, PUF, 1993. 
Fédération Européenne des journalistes, Étude sur la propriété des médias européens : menaces sur le paysage médiatique - Bruxelles avec le soutien de la Commission Européenne, 2002.

Gélard J. P., (dir.), Médias, mensonges et démocratie..., Rennes, Presses Universitaires de Rennes, 2004.

Leconte B., Thomas E. (dir.), Écrans et politique, Paris, L'Harmattan, 2004.

Martin-Lagardette J. L., Le guide de l'écriture journalistique, Paris, La Découverte, 2006.

Semprini A. (dir.), Analyser la communication 2, Paris, L'Harmattan, 2007.

Wolton D. (dir.), Événements mondiaux regards internationaux, Paris, CNRS éditions, 2006.

\section{Sites web}

La dichiarazione di indipendenza

Declaration of Independence of the "Corriere della Sera"

Journalists' Code of the Corriere della Sera

Lo statuto dei giornalisti del "Corriere della Sera"

Corriere della sera, storia di un grande quotidiano

RCS MediaGroup

Franco Venturini, editorialista del "Corriere della Sera" ...

\section{NOTES}

1. Voir à titre d'exemple: Agence Russe d'information Novosti «Poutine-Berlusconi : une rencontre entre "vieux amis" en Sardaigne ", 18 avril 2008.

La Repubblica "E a San Pietroburgo arriva Berlusconi per un abbraccio all' "amico Vladimir" - Il leader della Cdl incontra il presidente russo che ha sempre difeso anche sulla Cecenia - la visita », 18 avril 2008.

2. Les gouvernements italien et russe se rencontrent de façon alternée en Russie et en Italie pour développer les relations entre les deux pays. Le dernier sommet italo-russe s'est déroulé en Italie (13-17 mars 2007) et notamment à Bari. Depuis 2004 un « Foro di dialogo italo-russo delle società civili (Forum de dialogue italo-russe des sociétés civiles)» a été institué pour favoriser la compréhension réciproque pour ce qui est de la société civile et pour promouvoir les relations entre les deux pays. Cette institution prévoit plusieurs rencontres bilatérales annuelles. La dernière en date s'est déroulée à Moscou, le 2 avril 2008. [En ligne].

3. Le site du Ministère des affaires étrangères italien

4. F. Benvenuti, L'Europa vista dalla Russia. Avril-juin 2007. [Recherche].

5. "Putin attacca gli Usa: eccessivo ricorso alla forza ", Corriere della Sera, 11 février 2007. «Poutine met en accusation la politique américaine», Le Monde, 14 février 2007. "Vladimir Poutine dénonce l'unilatéralisme américain », Le Monde, 13 février 2007.

6. Le Corriere della Sera est le premier quotidien italien par diffusion. Il a été fondé en 1876 à Milan par Eugenio Torelli Viollier. Le 5-6 mars 1876, premier jour de publication du journal, Umberto Torelli Viollier déclara «Un fatto è un fatto e una parola non è che una parola (Un fait est un fait et un mot n'est qu'un mot) », «Ci piace essere obiettivi; di piace ricordarci che tu, pubblico (...) vuoi anzitutto essere informato con esattezza; ci piace serbare, di fronte a' nostri amici migliori, la nostra libertà di giudizio. (Nous aimons être objectifs ; nous aimons nous rappeler que toi, le public ( ...) tu veux avant tout être informé avec exactitude ; nous aimons garder devant nos meilleurs amis, notre liberté de jugement) ». Ces propos sont repris dans la déclaration d'indépendance du CDS publiée sur le site du journal. Le Corriere della Sera appartient au groupe Rcs Quotidiani spa qui est également propriétaire de Flammarion (à $100 \%$ ) et de Unidad Editorial qui publie El Mundo. Parmi les actionnaires de Rcs quotidiani spa, on compte la famille Agnelli, Benetton, Mediobanca. 
la Repubblica.it

Le Corriere della Sera

Étude sur la propriété des médias européens : menaces sur le paysage médiatique

7. Franco Venturini a été correspondant pour le Corriere della Sera à Moscou de 1986 à 1988 . Il a collaboré avec la $B B C$ ainsi qu'avec France Culture. Il parle 5 langues étrangères dont le russe et le français. [Source].

8. CDS est l'abréviation que nous allons utiliser pour Corriere della Sera.

9. Charaudeau P., Les médias et l'information. L'impossible transparence du discours, Bruxelles, De Boeck, 2005, p. 179-180.

10. La liste des journaux présents est proposée d'après l'ordre de leurs interventions lors de la conférence de presse.

11. Fabrizio Dragosei, Franco Venturini, « Putin: pronto a puntare i missili sull'Europa », Corriere della Sera,04/06/2007.

12. President of Russia - Official Web portal - Interview with Newspaper Journalists from G8 Member Countries - 4 juin 2007. [En ligne]

13. Martin-Lagardette J.-L., Le guide de l'écriture journalistique, La Découverte, Paris, p. 134-135, 2006.

14. Rauss F., La rédaction Web, p. 11, 2007.

15. Charaudeau P., Les médias et l'information. L'impossible transparence du discours, Bruxelles, De Boeck, p. 104, 2005.

16. Nous traduisons: "Tension avec les USA sur les armements, droits de l'homme, cas Litvinenko. "Je n'utilise pas un langage de lune de miel" ".

17. Arabyan M., Lire l'image, Paris, L'Harmattan, p. 14, 2000.

18. Martin-Lagardette J.-L., p. 144-145, 2006.

19. Nous traduisons : Novo-Ogaryovo (Russie) - Il est huit heures du soir, Vladimir Poutine est en retard car il est allé rendre visite à la veuve d'Eltsine. Les représentants des journaux invités par le Kremlin, un par pays du G8 qui va s'ouvrir ce mercredi en Allemagne l'attendent dans la datcha présidentielle de Novo-Ogaryovo, plongée dans un bois magnifique au-delà duquel poussent comme des champignons les résidences secondaires des nouveaux riches de Moscou. L'ambiance est détendue, fonctionnaires et gardes du corps jouent au billard pour tuer le temps. Mais dès que le Président arrive et que commence notre rencontre qui se déroulera par la suite à table, l'ambiance informelle et amicale qui règne dans la datcha va disparaître face à la dureté des paroles employées. Oui, répond Poutine à l'une de nos questions, les missiles nucléaires russes vont être de nouveau braqués sur les villes européennes et sur des cibles militaires en Europe si les Etats-Unis persévèrent à modifier l'équilibre stratégique en entraînant la Pologne et la République Tchèque dans la création d'un «bouclier » antimissile. Le message est fort, mais il convient de commencer par le commencement.

20. Texte source: And I am confident that an open, honest discussion between partners on all of these problems - no matter how difficult they are to resolve - will be a useful discussion.

21. Texte source : I would like to thank you for the interest you have shown in our work. And I certainly do not have the audacity or the responsibility of speaking for all my G8 colleagues. But I am ready to explain in more detail Russia's position on issues that you think are of interest to the public. That was everything I wanted to say at the outset and I will not waste time in a monologue. I am listening to you. Let's start working.

22. Ordre des sujets traités dans les questions de la conférence de presse d'après le texte source : fin de l'amitié Russie-Occident, bouclier USA en Europe orientale, missiles russes à Cuba, politique asiatique de la Russie, affaire Litvinenko, BP Shell, course à l'armement, traité INF et traité CFE, évolution des relations entre la France et la Russie avec le nouveau président français, Gazprom, retrait de la Russie du G8, souhait d'un monde multipolaire, Poutine considére comme dernier « pure démocrate » par Schroeder, Russie totalitaite, relations avec A. Merkel, législation 
concernant exportation de produits biologiques russes, relations Russie-Asie, avis de la Russie sur la candidature de Gordon Brown comme premier ministre, hypothèse sur succésseur de Poutine, bilan de son mandat présidentiel, ses projets pour l'après-élections, propositions de politique étrangère russe pour Iran et Kosovo, investissements des compagnies russes dans les entreprises européennes, Aeroflot-Alitalia, mauvaise image des entreprises russes en Europe, craintes concernant l'influence politique de la Russie, BP contrôlé à 51 \% par l'Etat, héritage politique, avenir «des hommes de Poutine » à la fin de son mandat, son épouse et sa famille, durée du mandat présidentiel, situation de transition en Russie, situation de la presse et des médias en Russie, exportation de crabes, manifestations réprimées, arrestations en Allemagne des opposants au G8, Union eurasienne, réaction de la Russie à intégration éventuelle de l'Ukraine dans l'UE et opinion de la Russie sur le fonctionnement de l'OTAN.

23. Nous avons ici souligné les passages «tronqués » dans le texte italien. Nous traduisons : « Si nous exprimons nos opinions ouvertement, honnêtement et franchement, cela ne signifie pas que nous cherchons l'affrontement. En outre, je suis profondément convaincu que si nous étions capables de rétablir un dialogue honnête ainsi que les capacités de trouver des compromis dans l'arène internationale, tous en tireraient profit. "

24. Passage tronqué dans le texte italien. Nous traduisons : «Je voudrais être compris ».

25. Idem. Nous traduisons: "C'est pour cette raison que nous ne voulons pas d'affrontement; nous voulons le dialogue. Toutefois, nous voulons un dialogue où l'équité des intérêts des deux parties en jeu soit reconnue. »

26. Ici il s'agit d'un ajout du quotidien italien. Nous traduisons : «Il est hors de question de parler de guerre froide. »

27. Martin-Lagardette J.-L., Le guide de l'écriture journalistique, La Découverte, Paris, p. 53-54, 2006.

28. Il s'agit d'une question en deux parties. Le journaliste a été interrompu par Poutine qui lui a fait remarquer que sa question était la réponse à la première question de l'hebdomadaire allemand.

29. Nous traduisons: "J'attire votre attention et celle de vos lecteurs sur le fait que pour la première fois dans l'histoire - et je souhaite le souligner - des éléments du système nucléaire des USA sont présents sur le continent européen. ».

30. Nous traduisons : «Est-ce qu'il vous arrive d'avoir la tentation de rendre la monnaie de la pièce à l'Amérique et d'installer des missiles russes à Cuba ou au Venezuela? »

\section{RÉSUMÉS}

Cet article se propose d'analyser les modalités mises en œuvre par les journalistes italiens du Corriere della Sera pour rapporter le discours de Vladimir Poutine. Le corpus de l'analyse est constitué par la retranscription de la conférence de presse tenue par Poutine le $1^{\text {er }}$ juin 2007 à la veille du G8 en Allemagne et par l'article relatant cet événement paru dans le Corriere della Sera du 4 juin. L'étude met à jour les différentes stratégies linguistiques et communicatives déployées par le quotidien italien pour mettre en scène l'événement et restructurer les propos tenus par le président russe en fonction de l'image de Poutine véhiculée dans la presse occidentale.

This article proposes to analyse the strategies deployed by journalists writing for the Italian daily Corriere della Sera as illustrated by the report of a speech delivered by Vladimir Putin on the eve of the G8 summit held in Germany in June 2007. The study focuses on the different linguistic and communicative strategies 
used by the Italian daily to depict the event and restructure the discourse of the Russian president in keeping with the image of the Vladimir Putin generally projected by the Western press.

\section{INDEX}

Keywords : Putin Vladimir, G8, press conference, Corriere Della Sera, Italy, Russia, reported speech, discourse analysis, image, self-censorship, manipulation

Mots-clés : Poutine Vladimir, G8, Corriere Della Sera, Italie, Russie, discours rapporté, analyse $\mathrm{du}$ discours, image, autocensure, manipulation

\section{AUTEUR}

ELISA ROSSI DANELZIK

Université Lyon 2

ILCEA / GREMUTS 